\title{
Improving the sexual health of young people (under 25 ) in high-risk populations: a systematic review and meta-analysis of behavioural and psychosocial interventions.
}

Ellie Brown ( $\nabla$ ellie.brown@unimelb.edu.au )

University of Melbourne https://orcid.org/0000-0002-1645-5443

Samantha L Lo Monaco

University of Melbourne

Brian O'Donoghue

The University of Melbourne

Elizabeth Hughes

University of Leeds

Melissa Graham

La Trobe University

Magenta Simmons

University of Melbourne

Richard Gray

La Trobe University

Research article

Keywords: sexual health, high-risk populations

Posted Date: April 3rd, 2021

DOI: https://doi.org/10.21203/rs.3.rs-380373/v1

License: (c) (i) This work is licensed under a Creative Commons Attribution 4.0 International License.

Read Full License 


\section{Abstract}

Background Ensuring young people experience good sexual health is a key public health concern. Yet, some vulnerable groups of young people are at higher risk of poor sexual health, and consequently require additional support to achieve good sexual health. Therefore, the aim of this systematic review was to identify and assess the evidence base for behavioural and psychosocial interventions to improve sexual health for young people with additional vulnerabilities.

Methods We searched for randomised controlled trials of interventions aimed at promoting sexual health, with any non-pharmacological comparator (e.g., waitlist control). Key outcomes of interest were indicators of sexual health (e.g., condom use, attitudes to contraception, knowledge of risk). Participants in eligible trials were under 25 years old and in a high-risk group (alcohol and other drug use; justiceinvolved; homeless; LGBTQI+; mental ill-health; ethnic minority, or out-of-home care). The final literature searches were performed on 16 September 2020, on MEDLINE, PsycINFO, EMBASE, CENTRAL, Web of Science, Scopus and clinical trial registries. Meta-analyses were conducted where possible.

Results Forty-seven papers from 46 trials of the 5213 identified met inclusion criteria, with all but one of the included trials conducted in North America. Three focused predominantly on AOD, six on juvenile justice, two on homelessness, five on young men who have sex with men (YMSM), 26 on ethnic minorities, two on mental ill-health, three on out-of-home care, however no trials were identified in LGBTQI + groups outside of YMSM. The 47 included papers had a combined total of 21,543 participants. The vast majority (26/46) of trials were conducted with ethnic minority groups, with most of the interventions delivered as group therapy, and some involving parents and caregivers. Condom use was the most frequently reported outcome measure. In trials targeting ethnic minorities, the meta-analysis found a medium effect size $(0.62, p=0.0004)$ of the intervention on condom use.

Conclusions There remains a dearth of research undertaken outside of North America, and in high-risk groups other than ethnic minorities. Future interventions should address sexual health more broadly than just the absence of negative biological outcomes with LGBTQI+, homeless and mental ill-health populations targeted for such work.

This review was registered at Prospero (ref. 149810) and at osf.io/ukva9.

\section{Background}

Good sexual health is broader than just being free from sexually acquired infections; rather it is defined as experiencing sexuality that is satisfying, positive, and respectful; as well as being free from exploitation and violence (1). As such, interconnecting biological and social factors play a part in how individuals experience sexual health.

The transition period between childhood and adulthood (typically considered between 10-25 years) is a time of significant biological, social, and psychological changes from the start of puberty to sexual 
maturation. During this time sex hormones increase, bodily changes occur, and a sense of self as a sexual being (including sexual identity) develops. Adolescents' experience newly emerging sexual desires and encounter opportunities to experiment with sexuality (2) however, emotional maturity has often not yet fully developed. As a result, adolescents and young people are more likely to engage in what can be considered "high risk" sexual activity. By this we mean activities that could lead to unintended pregnancy and/or sexually transmitted infections (STIs) such as condomless vaginal, anal, or oral sex or sex while under the influence of drugs or alcohol (3). This increased likelihood of engaging in high-risk sexual activity makes sexual health of critical concern within this age group (under 25). Data to support this concern include around $50 \%$ of new STIs (4), and $40 \%$ of new HIV infections (5) occurred among 15-24year-olds in the USA. Furthermore, when pregnancy occurs during adolescence rather than later in life, it is frequently associated with poor social outcomes (6).

In response to the need to address sexual health during this critical period, international agencies such as the United Nations Educational, Scientific and Cultural Organization (UNESCO) have declared that the delivery of sex education in schools should be mandatory (7). Whilst these school-based programs ensure the delivery of sex education to many, there are several groups amongst those aged under 25 that experience additional vulnerabilities that place them at higher risk of poor sexual health and may require a tailored approach that targets their specific needs.

When discussing "risky" groups it is important to acknowledge the impact this label can have on people, especially young people and those who are already marginalised by society for reasons like ethnicity or socioeconomic status (8). In this review we have chosen to search for interventions targeting young populations (under 25 years) who are underserved by the existing standard sexual education. This includes; LGBTQI + including young males/men who have sex with males/men (YMSM), individuals who use alcohol and other drugs (AOD) and/or experience mental ill-health, those in justice/prison or detention settings, ethnic minorities, and youth who are living in foster or out-of-home care $(\mathrm{OOHC})$.

These groups represent populations that have poorer sexual health than their peers. For example, YMSM account for approximately one third of new HIV infections among males/men who have sex with males/men (MSM) in the US (9), with many YMSM reportedly learning about anal sex through direct experience, in the absence of any appropriate sexual education (10), including discussion about the higher risks of STIs associated with anal sex. Young sexual minority females experience higher rates of forced sex and increased reporting of multiple partners (11), and young people with mental ill-health engage in higher rates of high-risk sexual behaviour and report a greater rate of unwanted pregnancy (12) than their peers. Youth who have alcohol or other substance use problems are more likely to have multiple partners, less likely to use condoms consistently, and therefore are at greater risk for STIs (13). Compared with youth who have never been incarcerated, young people in detention settings experience poorer mental and physical health, a higher incidence of STIs, more sexual partners, and higher rates of pregnancy (14). Ethnic minority youth experience disproportionately high rates of HIV infection, with over half of all new infections among YMSM in the US occurring in African-American youth, and a quarter in Hispanic or Latinx youth (15). Youth in foster care are more likely to be sexually active, and more likely to 
experience sexual debut before the age of 13 than those who have never been in foster care, with female adolescents more likely to experience earlier pregnancy (16).

Although there have been significant achievements in HIV prevention and treatment since the epidemic of the 1980s and 90s (for example Pre-exposure prophylaxis; PrEP), there has been markedly less advancement in sexual health promotion interventions for young people in vulnerable groups. In the Compendium of evidence-based and best practices for HIV prevention published by the CDC, only 10 of the included 59 interventions were developed for young people aged under 25 , despite the need to promote early intervention during this critical point in life (17).

Whilst systematic reviews of interventions for adults within specific high-risk populations have been completed (e.g., mental illness $(18,19)$, homelessness and drug use (20) justice involved individuals (21) and MSM (22)), the same has not for young people. The aim of the current systematic review is therefore to establish what behavioural interventions have been tested to improve sexual health among young people in at-risk populations, and how effective they are.

\section{Methods}

Before beginning the search, review databases (Cochrane, Prospero) were searched to confirm no similar reviews were already completed or in progress, and this review was registered on Prospero and at OSF (ref. 149810; osf.io/ukva9). We identified high-risk groups based on the Australian Department of Health's overview of the topic of youth at risk (23)

\section{Search strategy}

Literature searches were performed on MEDLINE, PsycINFO, Excerpta Medica Database (EMBASE), Cochrane Central Register of Controlled Trials (CENTRAL), Web of Science, and Scopus. Ongoing and unpublished trials were searched for at ClinicalTrials.gov, Australian and New Zealand Clinical Trials Registry (ANZCTR), and the World Health Organization's ISRCTN registry. The last search being performed on 16 September, 2020. Below is an example search strategy (as used for Ovid). Boolean operators are in bold.

1 (("safe sex" OR "sexual health" OR "sexual wellbeing" OR "sexual health promotion" OR "unsafe sex" OR "sexual risk taking" OR "sex* risk behavio*r" OR STI OR HIV OR chlamydia OR gonorrhoea OR hepatitis OR syphilis OR "acquired immunodeficiency syndrome" OR condom* OR "barrier contraception" OR "unwanted pregnancy" OR "pregnancy prevention") AND ("behavio* therapy" OR "cognitive therapy" OR "psychosocial intervention" OR "skills training" OR "sex education" OR "motivational interview*") AND (youth OR "young adult*" OR teenage* OR "high school student*" OR adolescen* OR "university student*")).ti,ab.

2 ("severe mental illness" OR "serious mental illness" OR "chronic mental illness" OR schizophrenia OR bipolar OR mania OR psychosis OR schizoaffective OR "major depressive disorder" OR MDD).ti,ab. 
3 (alcohol* OR binge OR ethanol OR drink* OR "drug use" OR "recreational drug*" OR "substance use").ti,ab.

4 ("juvenile justice" OR probation OR court* OR "law enforcement" OR "diversion program" OR "juvenile detention").ti,ab.

5 (homeless* OR "street youth" OR "runaway youth").ti,ab.

6 (gay OR lesbian* OR genderqueer OR transgender* OR homosexual* OR bisexual OR bicurious OR "female to male" OR "male to female" OR trans OR "men who have sex with men" OR MSM OR intersex OR LGBT OR LGBTQ OR LGBTQI OR "gender transition" OR "gender dysphoria" OR "gender identity" OR "same sex attracted" OR "same sex couple" OR "same sex couples" OR "sexual and gender minorities" OR "sexual orientation" OR "sexual preference" OR "trans wom\#n" OR "trans m\#n" OR "trans people" OR "women loving women" OR "women who have sex with women" OR WSW).ti,ab.

7 ("foster care" OR "group home*" OR "out of home care" OR "residential care").ti,ab.

8 ("minority group*" OR "ethnic minorit*" OR "ethnic group*" OR immigrant* OR "racial group*" OR black OR hispanic OR latin* OR asian OR indigenous OR "native American" OR aboriginal OR "first nations" OR african OR vietnamese OR "hawaii* native" OR asian OR indian OR inuit OR "pacific islander*" OR "american indian*").ti,ab.

111 AND (2 OR 3 OR 4 OR 5 OR 6 OR 7 OR 8) Inclusion criteria

Articles were included for review if they:

i. Included the following participant groups:

- Under 25 years old, any gender AND

- In a high-risk group

Alcohol and Other Drug (AOD) use

Justice involved youth

Homeless

LGBTQI

Mental ill-health

Ethnic minority

Out-of-home care (OOHC) 
ii. Were a randomised controlled trial whose arms $(\geq 2)$ are non-pharmacological interventions aimed at promoting sexual safety-taking behaviours and any non-pharmacological comparator (e.g., waitlist control)

iii. Reported sufficient data to satisfy PRISMA and Cochrane guidelines for inclusion in the review iv. Were published in English.

A study was ineligible if:

- it included participants older than 25 at the beginning of the trial

- the primary aim of the study was not to address sexual health

- it includes pharmacological treatment as a comparison arm

- there was a later publication of results from the same trial - the paper reporting the longest followup data was chosen

- if the intervention was focussed on abstinence, or purely targeted parents rather than the young person themselves.

\section{Data retrieval}

Data retrieved were uploaded to the referencing manager Mendeley. Mendeley was then used to remove duplicates from the review's sample.

\section{Data screening}

Titles and abstracts of papers were screened independently by two reviewers (S.L. and E.B.) following the eligibility criteria, using Covidence online review software. Papers that met eligibility criteria then had full articles screened. Discrepancies between the two reviewers were resolved by a third reviewer (R.G.).

\section{Data extraction}

A standardised form was used to extract data from the eligible trials and included: study citation, place of origin, setting, sample size, intervention type, dose, control, and findings. We noted in the table whether outcomes reported were based on participant knowledge and attitudes $(K+A)$, their self-reported behaviour (B), was an outcome that was biologically verified, i.e., STI infection or pregnancy (Bio), or related to sexual wellbeing, i.e., communication skills or relationship satisfaction (SW).

\section{Meta-analysis}

Where possible, data were combined from included trials using random effects meta-analysis. Specifically, for binary outcomes (e.g., STI incidence), data were pooled using odds ratios (ORs) and their associated $95 \%$ confidence intervals (Cls).

For outcomes measured on a continuous scale (e.g., attitudes and knowledge of STI/HIV), we used the mean difference (MD) or standardised mean difference (SMD) and their associated $95 \%$ Cls as 
appropriate. Specifically, where all trials included in a meta-analysis used the same measure, we used the MD. Where trials used different measures for the same outcome, we used the SMD.

Data from included trials were only combined where the population (e.g., the high-risk grouping) and outcome assessed (e.g., STI or pregnancy incidence, condom use, knowledge, or attitudes scales) were sufficiently similar. Where data from included trials could not be incorporated into a meta-analysis, we instead presented a narrative summary of the findings.

\section{Quality assessment of interventions}

All articles reviewed were subject to quality assessment using the Cochrane Risk of Bias tool. Assessments were performed independently by two reviewers (S.L. and E.B.) and with discrepancies being resolved by a third party where required.

\section{Results}

\section{Search results}

The final searches were performed on 16 September 2020, and 5213 papers were identified in total. The removal of 3490 duplicates left 1723 papers to be reviewed for eligibility, 1539 papers were removed following title and abstract screening, with 137 removed following full text screening. In total, 47 papers from 46 trials met our inclusion criteria with the PRISMA flow diagram (Fig. 1) showing details of reasons for exclusions, mainly being the wrong age range (for example, an age range that went above 25). The 47 included papers were spread over the high-risk groups as follows: three focused predominantly on AOD, six on juvenile justice, two on homelessness, five on YMSM, 26 on ethnic minorities, two on mental illhealth, three on out of home care, and finally no trials were identified in LGBTQI + groups outside of YMSM. The 46 included trials had a combined total of 21,543 participants and results are detailed in Table 1.

Nearly all (45/46) trials took place in the USA and tested interventions that were delivered in group settings, ranging from 60 minutes to eight hours, over one week to seven months. Six of the interventions were delivered at one time only. Some included trials had intersectional populations; where this was the case they are grouped according to the primary target population as stated by the authors, with secondary groups noted in relevant sections.

\section{Included trials}

\section{Alcohol and other drug (AOD) use}

Our review identified three papers describing interventions for youth with AOD-related issues. One was conducted inside a residential treatment facility (24), one in outpatient clinics (25), and one via text message (26) Only Letourneau (25) reported longer-term outcome data (over 12 months), focusing purely on behavioural outcomes. Their group intervention focused on reducing substance use and sexual riskbehaviours for young people who had been referred to a juvenile drug court. It incorporated caregiver 
involvement in a contingency management program, including elements of cognitive-behavioural therapy to help teens identify the antecedents of their risk behaviours. This intervention had no significant impact on sexual risk behaviours, with the authors highlighting the challenge of addressing common cooccurring 'problem behaviours' with one broad approach.

Five other papers identified in this review included young people with substance use problems as secondary groupings, given the commonality of comorbidities between these at-risk groups (Juvenile Justice and Homelessness groups) (27-31). These will be discussed under their primary grouping.

\section{Justice-involved youth}

We identified six papers reporting trials that targeted justice-involved youth $(27,28,31-34)$. Four of the six trials took place in detention centres or prisons, while one (32) was conducted in foster care homes adolescents had been placed in as part of their 'treatment'. Two of the trials were single gender only (one each for males (33) and females (32)), and two others also included content aimed at reducing drug and alcohol consumption $(27,28)$. The papers mostly reported behavioural outcomes such as condom use, pregnancy and STI incidence. One study (27) reported the reduction in STI incidence as a result of their sexual risk reduction group with additional content on alcohol and cannabis use. Kerr and colleagues (32), in a trial with girls aged 13-17, found multidimensional treatment foster care reduced the odds of pregnancy over the subsequent 24 months. The two other trials $(28,33)$ did not have a significant longterm impact on risky sexual behaviours despite post intervention effects on knowledge, attitudes and condom skills. No data were available to extract for meta-analyses within this high-risk group.

Four additional papers described three trials where some of the participants were involved with the justice system $(25,35-37)$.

\section{Homelessness}

Two papers focusing on homeless youth are included in this review; both were conducted at drop-in centres providing other services for homeless youth, and also included content to reduce AOD use (29, $30)$. Both trials reported significant findings when an unplanned post-hoc analysis was conducted. Slesnick et al. (29) found their intervention only had a significant impact on condom usage when age was factored into the analysis: intervention group participants aged 14-18 used condoms more frequently at follow up than control group youths aged 19-22. While the intervention used by Tucker and colleagues (30) failed to have an impact on knowledge and attitudes, it did decrease unprotected sexual acts for participants with multiple sexual partners.

\section{LGBTIQ+ (including YMSM)}

Five papers focused on YMSM (38-42). Two trials were conducted remotely, one of them online (40) and one via text message (41) as novel approaches to engaging this potentially hard to reach population. The remaining trials occurred in HIV clinics and LGBTIQ + community health centres. One occurred in Thailand (42). All five trials reported behavioural outcomes, typically rates of engagement in protected sex and the 
number of sexual partners. Overall, results were mixed with some, but not all, trials reporting a significant improvement in sexual health behaviours for participants who took part in an intervention. For example, Chen et al.'s (38) intervention increased participant's likelihood of using condoms $(p<.001)$ and Rongkavilit et al's (42) intervention reduced frequency of engaging in anal sex $(p=.04)$ but did not significantly improve condom use. Two of the five trials $(39,40)$ also reported knowledge and attitude outcomes, however neither intervention produced significant changes in these outcomes.

\section{Mental ill-health}

Two papers targeting young people with mental illness were identified; one recruited from mental health outpatient clinics (43), and the other (44) recruited high school students with "emotional or behavioural problems". Both were three-arm trials and produced mixed results. In Brown et al 2017 (44), the HIV prevention plus affect management (AM) intervention appeared to have more of an effect on sexual behaviours than a skills-based HIV prevention (SB), although both active interventions significantly improved HIV knowledge and condom attitudes at six months follow-up $(p<.05)$. There was no impact on engaging in sexual intercourse with concurrent substance use in either arm of the trial. Brown et al.'s (2014) (43) family-based HIV prevention and adolescent-only HIV prevention interventions both improved sexual behaviours at three months compared to control $(p<.05)$, but the family-based intervention also improved HIV knowledge and parent-teen sexual communication $(p<.01)$.

Two papers included in other high-risk groups also address youth with a history of abuse and/or mental ill-health $(36,45)$.

\section{Ethnic minorities}

There were 26 trials identified that tested a sexual health intervention targeting young people who were considered to be an ethnic minority. All were undertaken in the USA across a variety of settings, from sexual health clinics $(46,47)$ to community-based youth organisations $(48-50)$, and one provided in the context of a summer basketball camp for American Indian adolescents (51). Most (19/26) were delivered as group-based interventions, two as one-off interventions $(47,52)$, three interventions were delivered as one-to-one case management support (53-55) and one was a mass media intervention across different cities (56).

Fourteen of the interventions focused specifically on HIV prevention, four on HIV/STI prevention, two focused on preventing pregnancy, three had a focus on intervening at the family level, and the final three focused more broadly on reducing unprotected sex. Newer trials were less likely to focus specifically on HIV prevention, often recognising the need to address and measure sexual health more broadly than the prevention of one disease.

Two papers from other high-risk groups also included ethnic minorities, one targeting homelessness (30), and one for YMSM (40).

\section{Out of Home Care}


Three papers were identified from two trials that focused on delivering an intervention to young people in out of home care (35-37), with both recruiting a sample that were also involved in the juvenile justice system. The two trials tested intensive (at least twice weekly) group-based interventions delivered within the out of home care setting. Whilst the older trial (36) focused on HIV/AIDS prevention, (35)'s trial focused on pregnancy as well as HIV and STI prevention. Both trials found long-term (up to 12 months post intervention) significant change in knowledge and attitudes, but non-significant changes in sexual behaviours. (35) also assessed the sexual wellbeing concept of 'ability to communicate with partner' with the intervention group reporting a significantly higher ability over the 12 months follow up period.

\section{Meta-analysis}

Data were only available to undertake meta-analyses within one high-risk population; ethnic minorities. Data were pooled when available resulting in meta-analyses being completed on the following outcomes; STI/HIV/AIDS knowledge, condom use, frequency of unprotected sex and STI incidence).

\section{Knowledge of STI/HIV/AIDS}

Five papers provided sufficient data to produce a forest plot showing effect sizes for each study individually and pooled as an aggregate for the impact of a sexual health intervention on participants knowledge of STI/HIV/AIDS (Fig. 2). Standard mean differences are presented given inconsistency in outcome measure and end time points across trials. The pooled effect size of the five included trials was -0.34 ( $95 \% \mathrm{Cl}$ : -0.45 to -0.24$)$, suggesting a small effect size in favour of interventions. $I^{2}$ was $62 \%$, ( $p=$ $0.03)$.

\section{Condom use}

Data were available from a total of 12 trials to produce forest plots showing effect sizes for each study individually and pooled as aggregates for the impact of intervention on condom use (Figs. 3 and 4). Standard mean differences are presented for continuous data in Fig. 3, whilst odds ratios are presented in Fig. 4 for dichotomised data. Presentation of data on condom use was inconsistently presented, for example DiClemente et al (2004) (57) presented these data as the number of episodes of unprotected sex in last six months at 12 month follow up, whilst Jemmott et al (2005) (58) reported at the number of days of sex without condom in last three months and at 12 month follow up.

Pooled effect size for the trials that presented data as continuous variables was -0.05 ( $95 \% \mathrm{Cl}$ - -0.13 to $0.02)$, representing no effect $\left(I^{2}=0 \%, p=0.53\right)$. In contrast, the pooled odds ratio for trials presenting data as dichotomised variables was 0.62 ( $95 \% \mathrm{Cl}: 0.52$ to 0.74$)$; this suggests a medium size effect in favour of interventions $\left(I^{2}=74 \%, p=0.0004\right)$.

\section{Frequency of unprotected sex}

Data were available for a meta-analysis for a second behavioural outcome for ethnic minority groups; frequency of unprotected sex. Continuous data from four trials produced a pooled effect size of 0.19 ( $95 \%$ Cl: 0.10 to 0.28$)$; suggesting no effect $\left(I^{2}=0 \%, p=0.61\right)$, Fig. 5 . 


\section{STI incidence}

The biological outcome with data available for meta-analysis was STI incidence. The pooled odds ratio across six trials was 1.32 (95\% Cl: 1.07-1.64), representing a smaller likelihood of being diagnosed with an STI for participants in intervention conditions than those in controls (Fig. 6).

\section{Quality assessment of included trials}

The Cochrane risk of bias tool was used to assess the quality of all included papers, see Figs. 7 and 8 . Figure 7 shows that there was a high or unclear risk of bias for nearly all trials in the blinding of participants or personnel domain. The only domains where over half of the included trials were at a low risk of bias were incomplete outcome data and selective reporting.

\section{Discussion}

This systematic review of behavioural and psychosocial interventions to improve the sexual health of young people in high-risk groups identified a total of 47 trials reported between 1992 and 2018. Metaanalysis data suggest modest support for the efficacy of some interventions in increasing knowledge of HIV/AIDS, and decreasing incidence of STIs, although data were only available for interventions tailored to ethnic minority groups, which notably made up over half of the trials in this review. The large representation of ethnic minority samples is likely to be a reflection of the high rates at which the HIV pandemic continues to affect vulnerable groups in the USA (the target of this review), relative to the historically white majority (15). This is particularly evident within states exhibiting more pronounced racial health inequality, such as Mississippi, Louisiana, and Georgia. Therefore there is a need to test these interventions in other countries with different health systems and cultures, especially targeting less researched groups, for example homeless youth, those in OOHC, and non-YMSM LGBTQI + youth.

The most frequent outcomes reported in the included papers related to self-reported measures of behaviours that lowered risk of HIV and other STIs, such as frequency of unprotected sex, consistent condom use, and number of sexual partners. Change in knowledge and attitudes as a result of the intervention was also frequently measured. Most of the interventions were based on a model of health behaviour change, such as the Information-Motivation-Behaviour skills model $(59,60)$, which implies that whilst information is important for health behaviour change (i.e., knowledge), an individual's own motivation to change is also critical (i.e., attitudes). This review identified a number of trials $(33,35,36$, $61,62)$ that demonstrated that knowledge and attitudes toward sexual health and risk taking could be significantly improved in the intervention arm, but this did not translate into self-reported changes in behaviour. This was frequently attributed to the complex presentations of the participants that the interventions targeted, making the translation of knowledge into behaviour change an even greater challenge than it can typically be in public health interventions. Sexual health behaviour change requires assertiveness, planning, access to resources such as condoms and contraception, and engaging with partners that are non-coercive. For some groups this can be especially challenging when they face isolation, stigma, exploitation and abuse. Sexual health promotion interventions should take into account the need for empowerment, communication and social skills training, and access to appropriate 
resources and support. A minority (8/47) of the papers assessed outcomes relating to 'sexual wellbeing' such as communication skills with partners or parents. Doing so again recognises the need for sexual health promotion interventions for young people in high-risk groups to encompass healthy relationships, without solely focussing on the prevention of negative outcomes. The abilities to communicate needs and boundaries, and negotiate contraceptive use, are equally important in fostering long-term sexual health (63).

This review identified a substantial number (26) of trials that have been undertaken with young people of ethnic minorities in the USA. In contrast, only two trials were identified for three of the other populations: homelessness, $\mathrm{OOHC}$, and mental ill-health, and only three trials in AOD populations; highlighting the areas where considerable work is still required. Whilst five trials of YMSM were identified, none were found that focused on other LGBTQI + groups. There is a notable dearth of interventions for this group (excluding YMSM/MSM), particularly for trans youth (64).

The frequent intersectionality of trial populations - as evidenced by the multiple interventions addressing, for example, substance use in the context of homeless, justice-involved, or ethnic minority youth - could mean that young people struggle to access the support they need. When an individual is a member of several vulnerable groups, they are at risk of slipping through the cracks due to a lack of 'ownership' for the issue of their sexual health. Breaking down the siloed nature of physical health, mental health and social care services for these vulnerable individuals remains a key task at a clinical and policy level. In Australia, there has been an emphasis on the concept of a 'one stop shop' for youth mental health (65), although this model has faced criticism (66). Finding novel, effective ways to engage these groups, and supporting them to consider and address their sexual health at the critical period of adolescence needs to remain a priority of government, health and social services. Early, effective intervention for vulnerable youth could prevent the exacerbation of problematic economic and health disparities. In the UK, considerable success has been seen in public health interventions that target teen pregnancy, however there are still large disparities between certain groups (67).

\section{Limitations}

The results of this systematic review need to be considered in light of several limitations. Firstly, we excluded trials that utilised an age range of up to 29 years old, meaning we may have missed key interventions due to differences in definitions of 'young people'. Additionally, we used meta-analyses to compare trial data that often varied greatly in methodology. While we ensured all analysed variables were comparable, and used odds ratios to mitigate risk, the findings in favour of interventions need to be viewed with caution. In addition, raw data for use in meta-analyses were unavailable for several of the trials due to the way authors had chosen to present their data.

Ethnic minorities were only those from North America, suggesting that we cannot reliably extrapolate to other minority groupings such as Culturally and Linguistically Diverse (CALD) in Australia, or Black, Asian and Minority Ethnicities (BAME) in the UK. Many of these interventions were found acceptable by participants due to the tailoring of content to specific cultural groups. While this was effective to varying 
degrees for those included trials, it means that even the most promising interventions are likely needing cultural adaptation, co-produced with those who represent the voice of those cultures, before they could be tested in other settings and groups.

\section{Conclusions}

This systematic review found some support for sexual health promotion interventions that were tailored to specific high-risk populations of young people. Our challenge with being unable to perform metaanalyses across most of the data suggests that the field remains underdeveloped, and more randomised trials are required. Tackling the comorbidities commonly encountered by these populations is a challenge that requires consideration when developing or modifying interventions for these vulnerable populations. Attention should be paid not just to the health behaviour outcomes such as condomless sex, but also to the quality of the sexual relationships that young vulnerable people engage in, as well as the contexts in which sex occurs. This includes non-consensual sex and assault, exploitation, coercive controlling behaviours, lack of self-esteem and lack of social skills such as assertiveness, condom refusal in partners, and intimate partner violence.

Vulnerable young people experience significant challenges in their sexual health and wellbeing. As we have seen through teenage pregnancy interventions (67), it is likely that a good understanding of the specific issues faced must be factored into tailored and co-produced interventions that recognise the holistic nature of sexual wellbeing. Sexual wellbeing is not merely the prevention of disease, but also the empowerment of disenfranchised young people by focusing on building knowledge, a sense of selfworth, and promoting positive, rewarding sexual relationships that are free from abuse or coercion.

\section{Abbreviations}




\begin{tabular}{|l|l|}
\hline AIDS & Acquired ImmunoDeficiency Syndrome \\
\hline AM & Affect Management \\
\hline AOD & Alcohol and Other Drugs \\
\hline ANZCTR & Australian and New Zealand Clinical Trials Registry \\
\hline BAME & Asian and Minority Ethnicities \\
\hline CALD & Culturally and Linguistically Diverse Black \\
\hline CENTRAL & Cochrane Central Register of Controlled Trials \\
\hline CDC & Centre for Disease Control \\
\hline EMBASE & Excerpta Medica Database \\
\hline HIV & Human Immunodeficiency Virus \\
\hline ISRCTN & International Standard Randomised Controlled Trial Number \\
\hline LGBTQI & Lesbian, Gay, Bisexual, Transgender, Queer, Intersex \\
\hline MD & Mean Difference \\
\hline MSM & Men who have Sex with Men \\
\hline OOHC & Out Of Home Care \\
\hline PReP & PRe-exposure Prophylaxis \\
\hline PRISMA & Preferred Reporting Items for Systematic Reviews and Meta-Analyses \\
\hline SMD & Standardised Mean Difference \\
\hline STI & Sexually Transmitted Infections \\
\hline UNESCO & United Nations Educational, Scientific and Cultural Organization \\
\hline USA & United States of America \\
\hline WHO & World Health Organisation \\
\hline YMSM & Young Men who have Sex with Men \\
\hline
\end{tabular}

\section{Declarations}

Ethics approval and consent to participate

Not applicable. 
Consent for publication

Not applicable.

Availability of data and materials

All data used in this review, including meta-analysis data, are available upon request from the corresponding author.

Competing interests

There are no competing interests to declare.

\section{Funding}

There was no funding associated with this systematic review.

\section{Authors' contributions}

This review was conceived by EB and SL, with searches performed by SL and papers screened by SL, EB and RG. The paper was written by EB and SL, with contributions from BOD, EH, MG, MS, and RG. All authors read and approved the final manuscript.

Acknowledgements

Not applicable

\section{References}

1. World Health Organization. Defining sexual health: report of a technical consultation on sexual health, 28-31 January 2002, Geneva: World Health Organization; 2006.

2. Idele P, Gillespie A, Porth T, Suzuki C, Mahy M, Kasedde S, et al. Epidemiology of HIV and AIDS Among Adolescents: Current Status, Inequities, and Data Gaps. JAIDS Journal of Acquired Immune Deficiency Syndromes. 2014;66.

3. Chawla N, Sarkar S. Defining "High-risk Sexual Behavior" in the Context of Substance Use. Journal of Psychosexual Health. 2019;1(1):26-31.

4. Weinstock H, Berman S, Cates Jr W. Sexually transmitted diseases among American youth: incidence and prevalence estimates, 2000. Perspectives on sexual and reproductive health. 2004;36(1):6-10.

5. Satterwhite CL, Torrone E, Meites E, Dunne EF, Mahajan R, Ocfemia MCB, et al. Sexually Transmitted Infections Among US Women and Men: Prevalence and Incidence Estimates, 2008. Sexually Transmitted Diseases. 2013;40(3):187-93.

6. Cook SMC, Cameron ST. Social issues of teenage pregnancy. Obstetrics, Gynaecology \& Reproductive Medicine. 2015;25(9):243-8. 
7. Haberland N, Rogow D. Sexuality education: emerging trends in evidence and practice. Journal of adolescent health. 2015;56(1):S15-S21.

8. Shoveller JA, Johnson JL. Risky groups, risky behaviour, and risky persons: Dominating discourses on youth sexual health. Critical public health. 2006;16(1):47-60.

9. Centers for Disease Control Prevention. HIV surveillance in men who have sex with men (MSM). 2013. 2016.

10. Kubicek K, Beyer WJ, Weiss G, Iverson E, Kipke MD. In the dark: Young men's stories of sexual initiation in the absence of relevant sexual health information. Health Education \& Behavior. 2010;37(2):243-63.

11. Mojola SA, Everett B. STD and HIV risk factors among US young adults: Variations by gender, race, ethnicity and sexual orientation. Perspectives on Sexual and Reproductive Health. 2012;44(2):12533.

12. Adan Sanchez AY, McMillan E, Bhaduri A, Pehlivan N, Monson K, Badcock P, et al. High-risk sexual behaviour in young people with mental health disorders. Early Intervention in Psychiatry. 2018.

13. Cook RL, Comer DM, Wiesenfeld HC, Chang C-CH, Tarter R, Lave JR, et al. Alcohol and drug use and related disorders: An underrecognized health issue among adolescents and young adults attending sexually transmitted disease clinics. Sexually Transmitted Diseases. 2006;33(9):565-70.

14. Barnert ES, Perry R, Morris RE. Juvenile incarceration and health. Academic pediatrics. 2016;16(2):99109.

15. Centers for Disease Control and Prevention. Estimated HIV incidence and prevalence in the United States, 2010-2015. HIV Surveillance Supplemental Report http://www.cdc.gov/hiv/library/reports/surveillance/; 2018.

16. Gramkowski B, Kools S, Paul S, Boyer CB, Monasterio E, Robbins N. Health risk behavior of youth in foster care. Journal of child and adolescent psychiatric nursing. 2009;22(2):77-85.

17. Centres for Disease Control and Prevention. HIV/AIDS Prevention Research Synthesis Project. Compendium of Evidence-Based Interventions and Best Practices for HIV Prevention. Centers for Disease Control and Prevention. https://www.cdc.gov/hiv/research/interventionresearch/compendium/index.html2020 [updated 27 February.

18. Walsh C, McCann E, Gilbody S, Hughes E. Promoting HIV and sexual safety behaviour in people with severe mental illness: A systematic review of behavioural interventions. International journal of mental health nursing. 2014;23(4):344-54.

19. Pandor A, Kaltenthaler E, Higgins A, Lorimer K, Smith S, Wylie K, et al. Sexual health risk reduction interventions for people with severe mental illness: a systematic review. BMC Public Health. 2015;15(1):138.

20. Wright N, Walker J. Homelessness and drug use - a narrative systematic review of interventions to promote sexual health. AIDS Care. 2006;18(5):467-78. 
21. Underhill K, Dumont D, Operario D. HIV Prevention for Adults With Criminal Justice Involvement: A Systematic Review of HIV Risk-Reduction Interventions in Incarceration and Community Settings. American Journal of Public Health. 2014;104(11):e27-e53.

22. Herbst JH, Beeker C, Mathew A, McNally T, Passin WF, Kay LS, et al. The effectiveness of individual-, group-, and community-level HIV behavioral risk-reduction interventions for adult men who have sex with men: a systematic review. American journal of preventive medicine. 2007;32(4):38-67.

23. Australian Department of Health. Young people, risk and resilience: Learner's workbook. https://www1.health.gov.au/internet/publications/publishing.nsf/Content/drugtreat-pubs-front3-wktoc drugtreat-pubs-front3-wk-secb drugtreat-pubs-front3-wk-secb-2 drugtreat-pubs-front3-wk-secb2-1); 2004.

24. St. Lawrence J, Jefferson K, Alleyne E, Brasfield T. Comparison of Education Versus Behavioral Skills Training Interventions in Lowering Sexual HIV-Risk Behavior of Substance-Dependent Adolescents. Journal of Consulting and Clinical Psychology VO - 63. 1995(1):154-.

25. Letourneau EJ, McCart MR, Sheidow AJ, Mauro PM. First Evaluation of a Contingency Management Intervention Addressing Adolescent Substance Use and Sexual Risk Behaviors: risk Reduction Therapy for Adolescents. Journal of substance abuse treatment. 2017;72:56-65.

26. Suffoletto B, Akers A, McGinnis KA, Calabria J, Wiesenfeld HC, Clark DB. A Sex Risk Reduction TextMessage Program for Young Adult Females Discharged From the Emergency Department. Journal of Adolescent Health. 2013;53(3):387-93.

27. Bryan AD, Magnan RE, Gillman AS, Yeater EA, Feldstein Ewing SW, Kong AS, et al. Effect of including alcohol and cannabis content in a sexual risk-reduction intervention on the incidence of sexually transmitted infections in adolescents: A cluster randomized clinical trial. JAMA Pediatrics. 2018;172(4).

28. Bryan AD, Schmiege SJ, Broaddus MR. HIV risk reduction among detained adolescents: a randomized, controlled trial. Pediatrics. 2009;124(6 CC - Drugs and Alcohol CC - SR-BEHAVMED CC HIV/AIDS):e1180-8.

29. Slesnick N, Kang MJ. The impact of an integrated treatment on HIV risk behavior among homeless youth: a randomized controlled trial. Journal of behavioral medicine. 2008;31(1 CC - HIV/AIDS):4559.

30. Tucker JS, D'Amico EJ, Ewing BA, Miles JN, Pedersen ER. A group-based motivational interviewing brief intervention to reduce substance use and sexual risk behavior among homeless young adults. Journal of substance abuse treatment. 2017;76:20-7.

31. Tolou-Shams M, Houck C, Conrad SM, Tarantino N, Stein LAR, Brown LK. HIV Prevention for Juvenile Drug Court Offenders: A Randomized Controlled Trial Focusing on Affect Management. Journal of Correctional Health Care. 2011;17(3):226-32.

32. Kerr DCR, Leve LD, Chamberlain P. Pregnancy Rates Among Juvenile Justice Girls in Two Randomized Controlled Trials of Multidimensional Treatment Foster Care. Journal of Consulting and Clinical Psychology. 2009;77(3):588-93. 
33. St. Lawrence JS, Crosby RA, Belcher L, Yazdani N, Brasfield TL. Sexual Risk Reduction and Anger Management Interventions for Incarcerated Male Adolescents: A Randomized Controlled Trial of Two Interventions. JOURNAL OF SEX EDUCATION AND THERAPY VO - 24. 1999:9-.

34. Goldberg E, Millson P, Rivers S, Manning SJ, Leslie K, Read S, et al. A Human Immunodeficiency Virus Risk Reduction Intervention for Incarcerated Youth: A Randomized Controlled Trial. Journal of Adolescent Health. 2009;44(2):136-45.

35. Green J, Oman RF, Lu M, Clements-Nolle KD. Long-Term Improvements in Knowledge and Psychosocial Factors of a Teen Pregnancy Prevention Intervention Implemented in Group Homes. Journal of Adolescent Health. 2017;60(6):698-705.

36. Slonim-Nevo V, Auslander WF, Ozawa MN, Jung KG. The Long-Term Impact of AIDS-Preventive Interventions for Delinquent and Abused Adolescents. ADOLESCENCE VO - 31. 1996:409-.

37. Oman RF, Vesely SK, Green J, Clements-Nolle K, Lu M. Adolescent Pregnancy Prevention Among Youths Living in Group Care Homes: A Cluster Randomized Controlled Trial. American Journal of Public Health. 2018;108:S38-S44.

38. Chen X, Murphy DA, Naar-King S, Parsons JT. A clinic-based motivational intervention improves condom use among subgroups of youth living with HIV. Journal of Adolescent Health. 2011;49(2):193-8.

39. Hidalgo MA, Kuhns LM, Hotton AL, Johnson AK, Mustanski B, Garofalo R. The MyPEEPS Randomized Controlled Trial: A Pilot of Preliminary Efficacy, Feasibility, and Acceptability of a GroupLevel, HIV Risk Reduction Intervention for Young Men Who Have Sex with Men. Archives of Sexual Behavior VO - 44. 2015(2):475-.

40. Mustanski B, Garofalo R, Monahan C, Gratzer B, Andrews R. Feasibility, acceptability, and preliminary efficacy of an online HIV prevention program for diverse young men who have sex with men: the keep it up! intervention. AIDS and behavior. 2013;17(9 CC - HIV/AIDS CC - Consumers and Communication):2999-3012.

41. Ybarra ML, Liu W, Prescott TL, Phillips G, Mustanski B. The Effect of a Text Messaging Based HIV Prevention Program on Sexual Minority Male Youths: a National Evaluation of Information, Motivation and Behavioral Skills in a Randomized Controlled Trial of Guy2Guy. AIDS and behavior. 2018;22(10):3335-44.

42. Rongkavilit C, Wang B, Naar-King S, Bunupuradah T, Parsons JT, Panthong A, et al. Motivational interviewing targeting risky sex in HIV-positive young Thai men who have sex with men. Archives of sexual behavior. 2015;44(2 CC - HIV/AIDS):329-40.

43. Brown L, M. D., Wendy Hadley PD, Geri R. Donenberg PD, Ralph J. DiClemente PD, Celia Lescano PD, Delia M. Lang PD, et al. Project STYLE: A Multisite RCT for HIV Prevention Among Youths in Mental Health Treatment. Psychiatric Services. 2014;65(3):338-44.

44. Brown LK, Whiteley L, Houck CD, Craker LK, Lowery A, Beausoleil N, et al. The Role of Affect Management for HIV Risk Reduction for Youth in Alternative Schools. Journal of the American Academy of Child \& Adolescent Psychiatry. 2017;56(6):524-31. 
45. Champion JD, Collins JL. Comparison of a theory-based (AIDS Risk Reduction Model) cognitive behavioral intervention versus enhanced counseling for abused ethnic minority adolescent women on infection with sexually transmitted infection: Results of a randomized controlled trial. International Journal of Nursing Studies. 2012;49(2):138-50.

46. DiClemente RJ, Wingood GM, Rose ES, Sales JM, Lang DL, Caliendo AM, et al. Efficacy of sexually transmitted disease/human immunodeficiency virus sexual risk-reduction intervention for African American adolescent females seeking sexual health services: A randomized controlled trial. Archives of Pediatrics and Adolescent Medicine. 2009;163(12):1112-21.

47. Crosby RA, Charnigo RJ, Salazar LF, Pasternak R, Terrell IW, Ricks J, et al. Enhancing Condom Use Among Black Male Youths: A Randomized Controlled Trial. American Journal of Public Health. 2014;104(11):2219-25.

48. Dilorio C, McCarty F, Resnicow K, Lehr S, Denzmore P. REAL men: a group-randomized trial of an HIV prevention intervention for adolescent boys. American Journal of Public Health. 2007;97(6):1084-9.

49. Dilorio C, Resnicow K, McCarty F, De AK, Dudley WN, Wang DT, et al. Keepin' It R.E.A.L.!: Results of a Mother-Adolescent HIV Prevention Program. NURSING RESEARCH -NEW YORK THEN HAGERSTOWNVO - 55. 2006:43-.

50. Jemmott JB, Ls J, Gt F, Kh M. Effectiveness of an HIV/STD risk-reduction intervention for adolescents when implemented by community-based organizations: a cluster-randomized controlled trial. American Journal of Public Health. 2010;100(4):720-6.

51. Tingey L, Mullany B, Chambers R, Hastings R, Lee A, Parker A, et al. Respecting the circle of life: one year outcomes from a randomized controlled comparison of an HIV risk reduction intervention for American Indian adolescents. AIDS Care. 2015;27(9):1087-97.

52. St. Lawrence JS, Brasfield TL, Jefferson KW, Alleyne E, O'Bannon lii RE, Shirley A. Cognitivebehavioral intervention to reduce African American adolescents' risk for HIV infection. Journal of Consulting and Clinical Psychology VO - 63. 1995(2):221-.

53. Barnet B, Liu J, DeVoe M, Duggan AK, Gold MA, Pecukonis E. Motivational intervention to reduce rapid subsequent births to adolescent mothers: a community-based randomized trial. Annals of family medicine. 2009;7(5):436-45.

54. Sieving RE, McRee A-L, Secor-Turner M, Garwick AW, Bearinger LH, Beckman KJ, et al. Prime Time: Long-Term Sexual Health Outcomes Of a Clinic-Linked Intervention. Perspectives on Sexual and Reproductive Health. 2014;46(2):91-100.

55. Smith ML, Wilson KL, Bergeron CD. Condom Use for Sexually Transmitted Infection Prevention Among Hispanic Teenage Mothers: A Community-Based Randomized Trial. Journal of Women's Health (15409996). 2020;29(4):534-40.

56. Sznitman S, Vanable PA, Carey MP, Hennessy M, Brown LK, Valois RF, et al. Using Culturally Sensitive Media Messages to Reduce HIV-Associated Sexual Behavior in High-Risk African American Adolescents: Results From a Randomized Trial. Journal of Adolescent Health. 2011;49(3):244-51. 
57. DiClemente RJ, Wingood GM, Harrington KF, Lang DL, Davies SL, Hook EW, et al. Efficacy of an HIV Prevention Intervention for African American Adolescent Girls: A Randomized Controlled Trial. JOURNAL- AMERICAN MEDICAL ASSOCIATION VO - 292. 2004:171-.

58. Jemmott JB, Jemmott LS, Braverman PK, Fong GT. HIV/STD risk reduction interventions for African American and Latino adolescent girls at an adolescent medicine clinic: a randomized controlled trial. Archives of Pediatrics \& Adolescent Medicine VO - 159. 2005:440-.

59. John SA, Walsh JL, Weinhardt LS. The Information-Motivation-Behavioral Skills Model Revisited: A Network-Perspective Structural Equation Model Within a Public Sexually Transmitted Infection Clinic Sample of Hazardous Alcohol Users. AIDS and behavior. 2017;21(4):1208-18.

60. Fisher EB, Fitzgibbon ML, Glasgow RE, Haire-Joshu D, Hayman LL, Kaplan RM, et al. Behavior Matters. American Journal of Preventive Medicine. 2011;40(5):e15-e30.

61. Jemmott JB, Jemmott LS, Fong GT. Reductions in HIV Risk-Associated Sexual Behaviors among Black Male Adolescents: Effects of an AIDS Prevention Intervention. American Journal of Public Health. 1992;82(3):372-7.

62. Kipke M, Boyer $C$, Hein K. An evaluation of an AIDS risk reduction education and skills training (Arrest) program. Journal of Adolescent Health. 1993;14(7):533-9.

63. Horwood J, Morden A, Bailey JE, Pathak N, Feder G. Assessing for domestic violence in sexual health environments: a qualitative study. Sexually Transmitted Infections. 2018;94(2):88-92.

64. Hibbert MP, Wolton A, Weeks H, Ross M, Brett CE, Porcellato LA, et al. Psychosocial and sexual factors associated with recent sexual health clinic attendance and HIV testing among trans people in the UK. BMJ Sexual \&amp; Reproductive Health. 2020;46(2):116-25.

65. Rickwood D, Paraskakis M, Quin D, Hobbs N, Ryall V, Trethowan J, et al. Australia's innovation in youth mental health care: The headspace centre model. Early intervention in psychiatry. 2019;13(1):159-66.

66. Jorm AF. Headspace: The gap between the evidence and the arguments. Australian \& New Zealand Journal of Psychiatry. 2016;50(3):195-6.

67. Public Health England. Teenage pregnancy prevention framework; supporting young people to prevent unplanned pregnancy and develop healthy relationships. https://www.gov.uk/government/publications/teenage-pregnancy-prevention-framework; 2018.

\section{Table 1}

Due to technical limitations Table 1 is available as a download in the Supplementary Files.

\section{Figures}




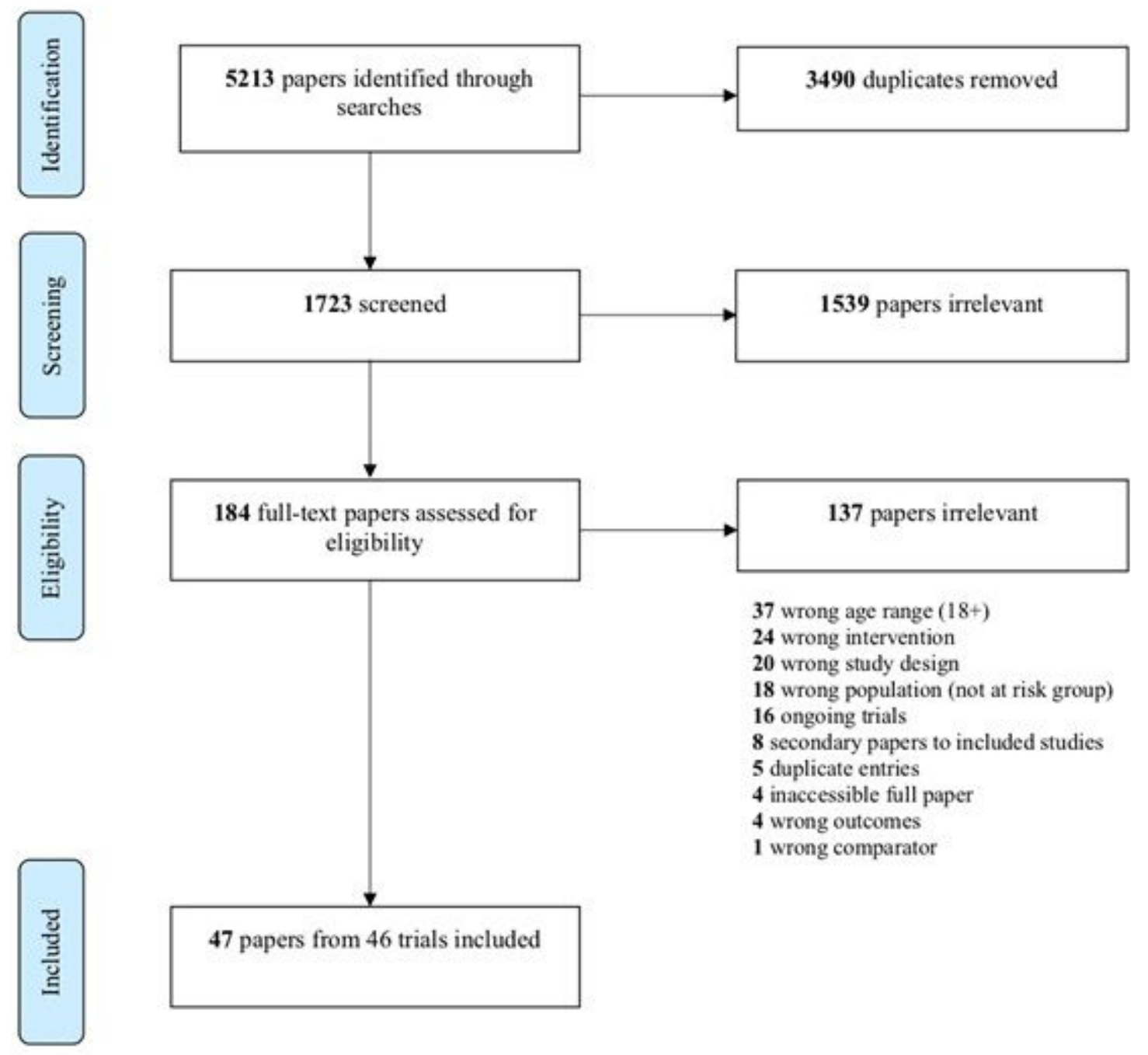

Figure 1

PRISMA flow diagram

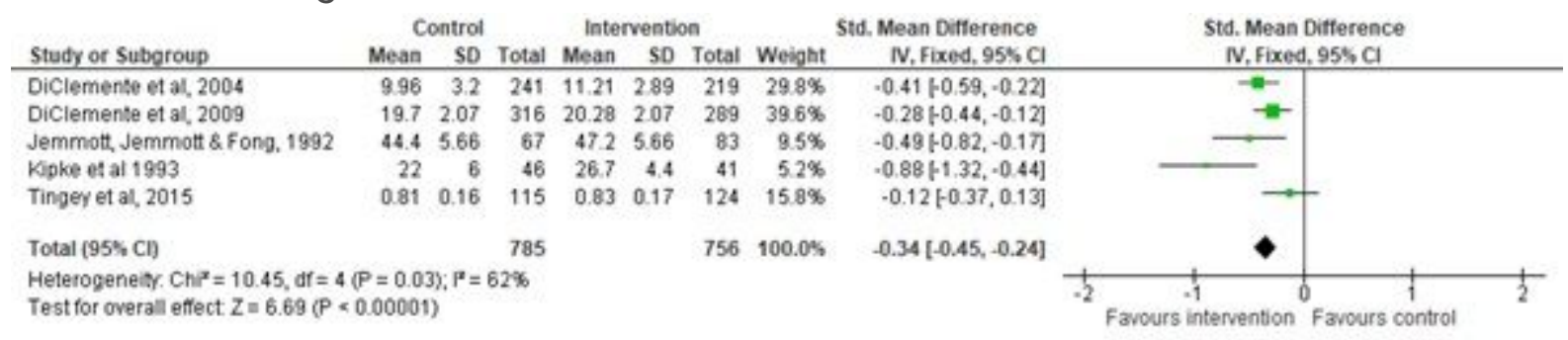

Figure 2

Forest plot of STI/HIV/AIDS knowledge in youth ethnic minorities. 


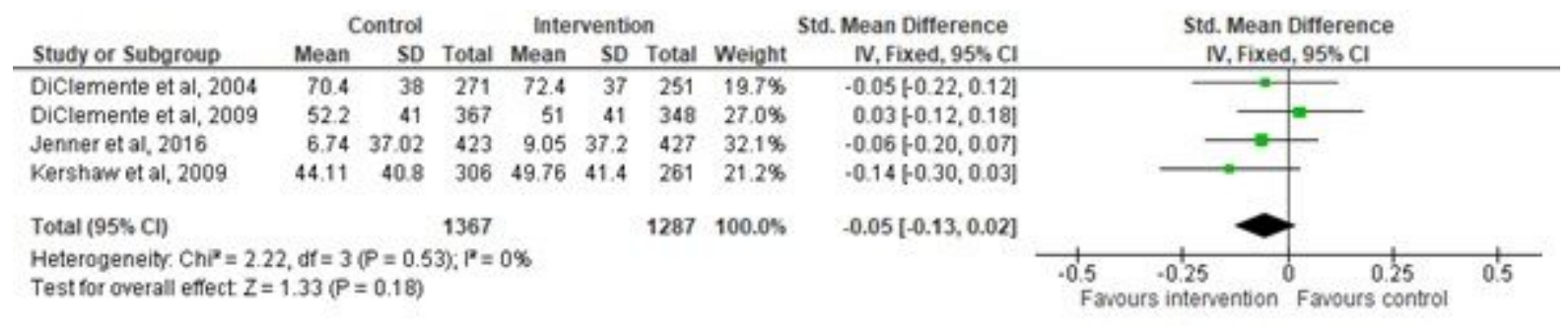

Figure 3

Forest plot of condom use measured using continuous variables in youth ethnic minorities

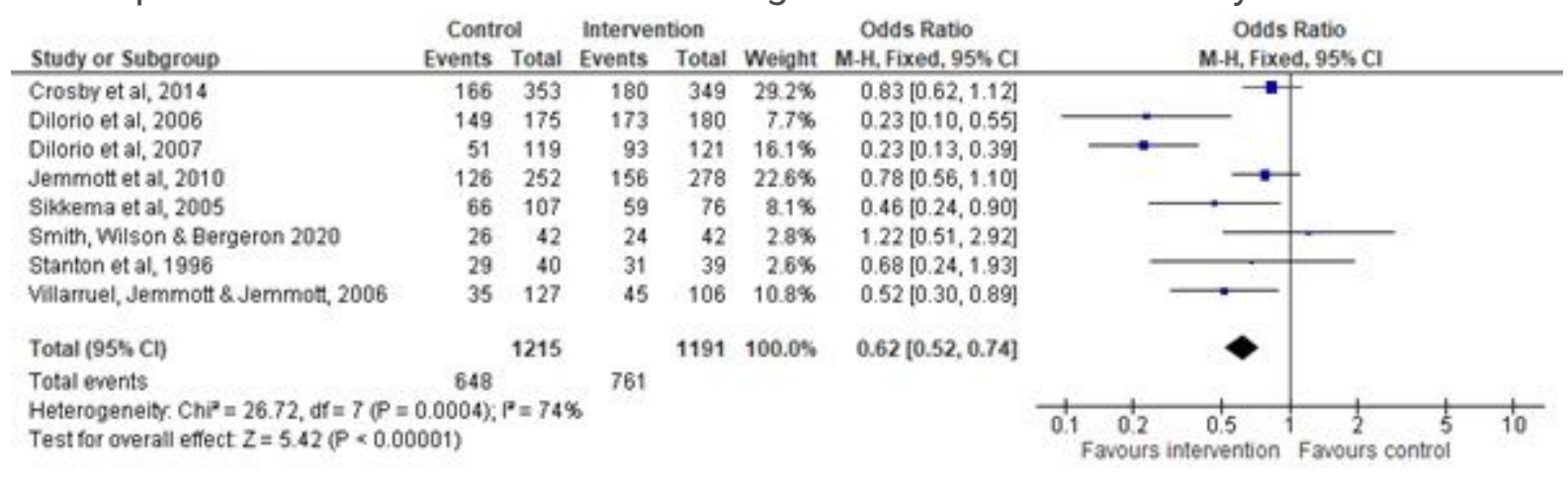

\section{Figure 4}

Forest plot of condom use measured using dichotomised variables in youth ethnic minorities

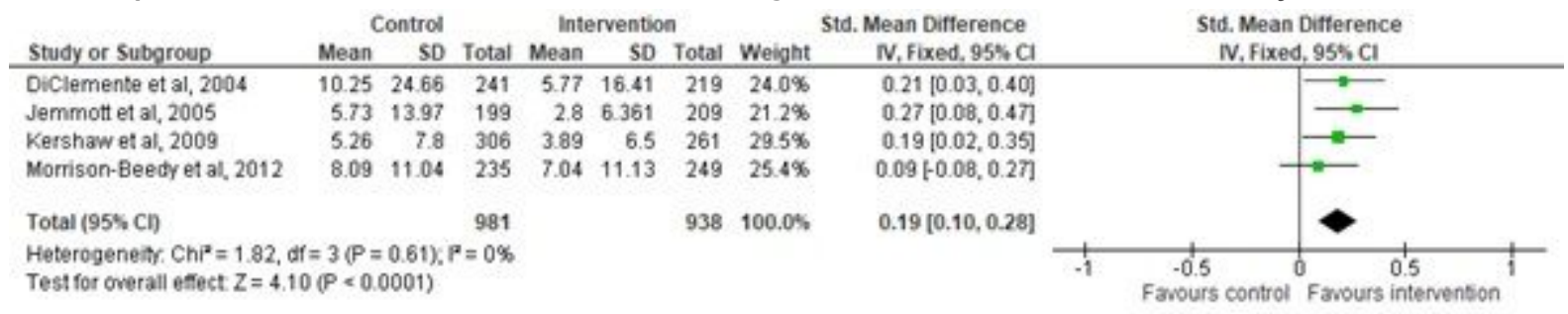

Figure 5

Forest plot of frequency of unprotected sex in youth ethnic minorities

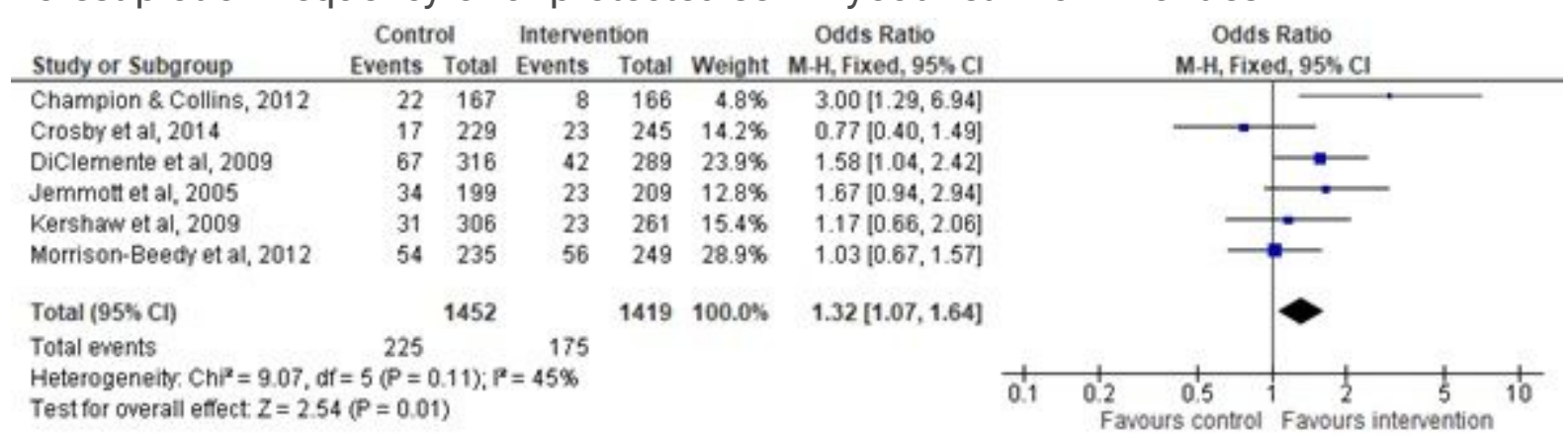

Figure 6

Forest plot of STI incidence in youth ethnic minorities. 


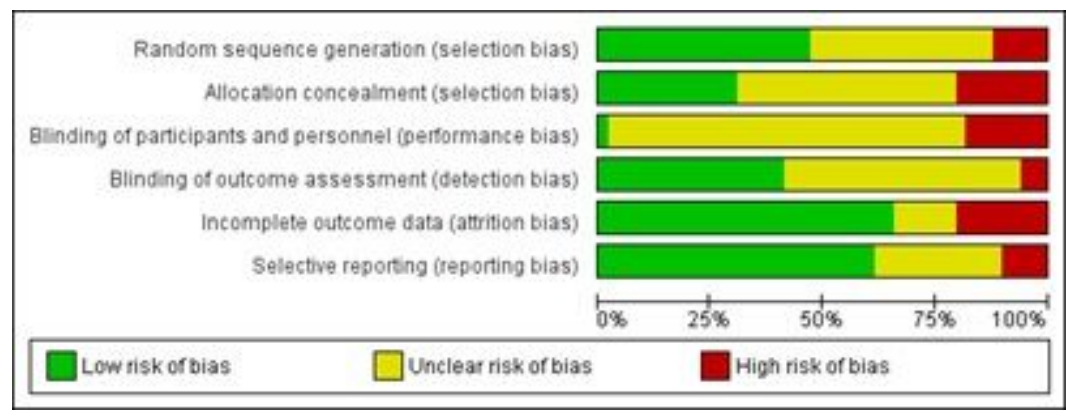

\section{Figure 7}

Summary of risk of bias assessments of included trials. 


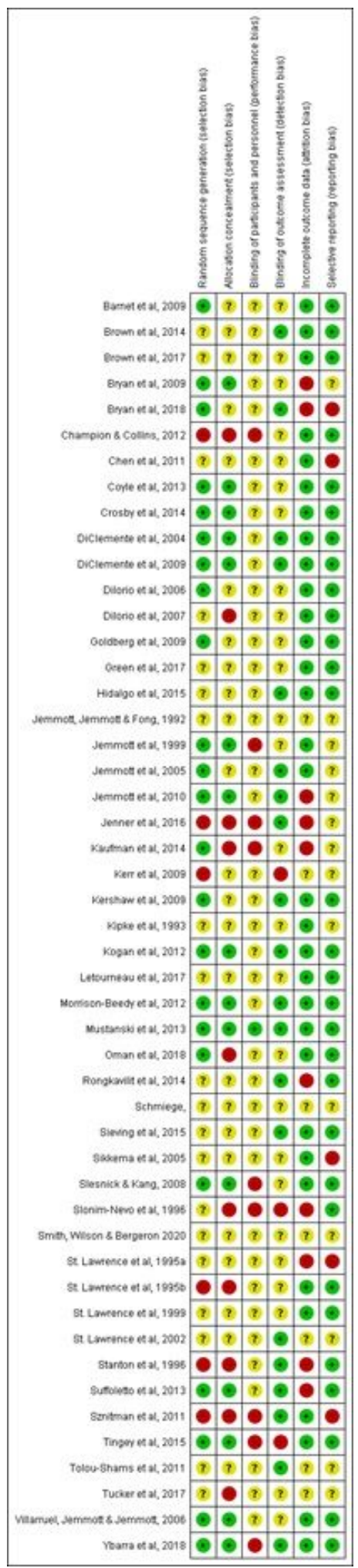

\section{Figure 8}

Detailed risk of bias assessments for each paper.

\section{Supplementary Files}

This is a list of supplementary files associated with this preprint. Click to download. 
- PRISMA2009checklist30.12.20.docx

Page 25/25 